DOE/ER/ 14100--1

DE92 004077

\title{
Hydrodynamic Instabilities and Coherent Structures
}

\section{Progress Report}

January 1, 1991 - December 31, 1991

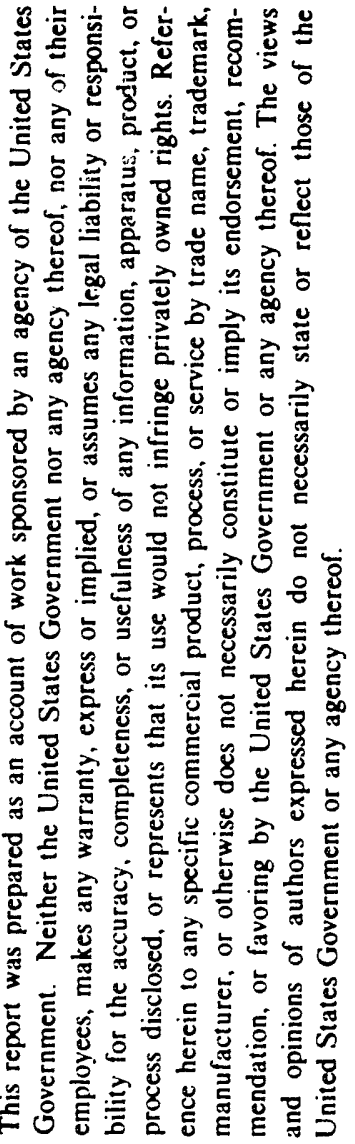

\author{
Alexander Frenkel, Principal Investigator
}

August 1991

Prepared for the U. S. Department of Energy

Under Grant Number DE-FG05-90ER14100 
The University of Alabama.

Report on Activities under the U.S. Department of Energy Grant

$$
\text { \# DE-FG05-90ER14100 }
$$

"HYDRODYNAMIC INSTABILITIES AND COHERENT STRUCTURES"

January 1, 1991---December 31, 1991

During the period under review significant progress has been made along the principal lines of the project. Specifically:

1. A computer code has been created (in collaboration with Professor R. Pelz, Rutgers University) and tested for the purpose of finding the most unstable normal modes of magnetic field which can grow due to the (kinematic) dynamo action of the $A B C-f l o w$ (of an electrically conducting fluid).

This periodic flow has chaotic stream lines and therefore has long been considered a good candidate for the fast dynamo action. V.I. Arnol'd and E.I. Korkina, and also D.J. Galloway and U. Frisch, found numerically that there is an "island" of stability on the axis of magnetic Reynolds numbers. However, as was pointed out by the PI (A. Frenkel, Physics of Fluids A 3 (1991) 1718-1728; also, see the proposal for the present project), those researchers have considered only an infinitely small part of all possible normal modes: they left out all the modes which do not have the periodicity of the basic ABC flow.

Our preliminary computations of the latter normal modes indicate an interesting result: the above mentioned "island of stability" disappears. In general, the magnetic field grows faster when it contains these new modes.

We are using CRAY XMP/24 of Alabama Supercomputer Network for this work. (A graduate research assistant of the PI takes a part in this investigation.)

2. A computer code has been created and tested (in collaboration with Dr. G. Rudenko, USSR) to investigate the linear stability of the time-periodic kolmogorov flow (see the PI's paper referred to above). The computations converge very fast even for arbitrary large Reynolds numbers.

The ideas on which the code is based seem to be generalizable to time dependent periodic flows of higher spatial

dimensionality. The investigation of such flows and the convergence of the results obtained by the Galerkin type truncations of the infinite eigen-problem for the quasi-normal modes (introduced in the PI's paper referred to above) is in the research plan of the present project. The PI considers inviting Dr. Rudenko as a Postdoctoral visitor for the 
accelerated continuation of this work (subject to availability of funds in the grant budget; see (b) on p. 4 below).

3. We have studied (in collaboration with professor G. Sivashinsky, Tel-Aviv University) the stability of the timeperiodic triangular-eddy flow (as was suggested in the proposal for the present project). We found that the oscillations of the basic flow at small Reynolds numbers can decrease the effective viscosity responsible for the dissipation of large-scale perturbations.

Moreover, by using his quasi-normal mode approach and the first Galerkin truncation of the infinite algebraic eigenproblem, the PI has found that the viscosity can even become negative at higher Reynolds numbers and in a finite window of oscillation frequencies. This seems to indicate that unisotropy is not necessary for the large-scale instability, at least in the case of the time-dependent basic flow. This dispels some earlier beliefs and partially answers the question posed in a recent work by B. Dubrulle and U. Frisch (Phys. Rev. A 43 (1.991) 5355).

These results are now in preparation for publication. However, the question of convergence of the Galerkin truncations (posed in our proposal and in the PI's paper referred to above) calls for the investigation of higher Galerkin truncations. This can be done numerically only, and we are planning this work for the near future. The experience with other periodic flows (admittedly, time-independent ones, since the studies of time-dependent flows started just recently) suggests that the conclusions based on the first Galerkin truncation will be justified by the investigation of the higher truncations.

4. The nonlinear theory of strongly undulating thin films flowing down vertical fibers has been developed by the PI.

Although the flow under consideration has many similarities with the core-annular flow on which the PI's proposal for this project was focused, the free-surface flow was not planned for investigation there. However, a very interesting experimental investigation by Quere has been published recently (Europhys. Lett. 13 (1990) 721; Science 249 (1990) 1256). The film flow studies have a long history. These flows has been studied extensively, since they exhibit a variety of intriguing phenomena on one hand and are important in many industries on the other hand. Nevertheless, Quere (apparently guided by the idea - suggested earlier by the PI for a core-annular case that, when a basic state is not stagnant, the Rayleigh instability of annular films can be saturated by a nonlinear mechanism) was able to reveal a quantity which was never considered before - the critical thickness of the film. This critical thickness is the border point between the thicker basic films which are ruptured by the instability and thinner 
films which, because of the nonlinear saturation of the instability, develop only small ripples on their surface. Quere's measurements resulted in a classical-type law: the critical film thickness is proportional to the cube of the fiber radius. Also, the critical thickness turned out to be independent of viscosity. Quere found that these dependences follow from a nonlinear equation of interface evolution which can be derived in analogy with our earlier derivation of a similar equation for the core-annular case. However, both derivations imply a film which develops only small ripples. The case of the critical film is different: the amplitudes of film undulations are as large as the (critical) film thickness.

In the work of the PI reported here, a new equation is derived which does include the critical case, and Quere's cubic law and viscosity-independence law are obtained as consequences of this new nonlinear equation. The Kuramoto-sivashinsky type equation of Quere is a particular asymptotic limit of the new equation. The multiparameter approach to the nonlinear problems of film flows (developed earlier by the PI), on which our derivation of the theory is based, lead to relatively nonrestrictive sufficient conditions of applicability: these conditions confine the values of flow parameters to a full (3dimensional) domain in the 3-dimensional space of basic parameters (whose values enumerate the basic flows). The mechanism of nonlinear saturation in the free-surface film has some interesting differences from the core-annular case.

This work has been submitted for publication.

5. The multiparameter approach which was first formulated briefly by the PI in 1988 (Proc. Of the 6th Symp. On Energy Engineering Sci. (Argonne, Illinois) p. 100) is at the focus of discussion in our work on the nonlinear rupture of a van der Waals film (Proc of the 9 th Symp. on Energy Eng. Sci., Argonne, Illinois 1991, p. 185).

This problem was considered before by $M$. Williams and $s$. Davis. They used a single-parameter technique. Because of this, the evolution equation they derived could be justified for merely a segment of a line in the space of basic parameters. $K$. Chen and $D$. Joseph noted recently that realistic values of the basic parameters, that is of the Hamacker constant (which characterizes the molecular forces responsible for the film break-up) and the surface tension coefficient, are outside the domain of applicability which is implied by the derivation of williams and Davis.

In our work, however, we show that a multiparameter derivation is possible which expands the applicability of the evolution equation to a full-dimensional domain in the space of basic parameters. The realistic van der waals films fall well inside this expanded domain. Thus, the theory does describe the nonlinear break up of the films which can be observed in experiments. 
There are just two independent parameters in this problem, which makes it especially suitable for demonstrating the tech rique of the multiparameter approach.

6. An interfacial evolution equation has been derived by the PI for the core-annular flow in a vertical pipe, similar to the flow which was studied in experiments of $D$. Joseph and his collaborators.

For the case of small Reynolds numbers in the annular film, the equation differs little from the one derived by the PI in the paper of 1988 referred to above (this latter equation was discussed recently by $K$. Chen and D. Joseph, JFM 227, 1991, 587, who called it the Kuramoto-sivashinsky-Frenkel equation. They made some critical remarks wich were answered by the PI in his 1991 work referred to above. It was noted there that some of those remarks are addressed rather to the singleparameter re-derivation of the equation by Papageorgiou et al., referred to below).

The new equation can be made essentially the same as the equation of the 1988 paper by a suitable choice of the reference frame. We are planning to publish this work because the Proceedings in which our previous related work was published is not readily available to a reader. (Although the results of our previous work has been published by $D$. Papageorgiou et al. in Phys. Fluids $A(2,1990$, p. 340), they were rederived there in a single-parameter approach which we believe to be inferior to our multiparameter approach for the reasons mentioned above.)

The numerical simulations of this interface equation (which contains both dispersive and dissipative effects and therefore is expected to lead to nonlinear periodic waves instead of chaotic oscillations typical for a related Kuramoto-sivashinsky equation) are desirable (as was suggested in the proposal for this project). The numerical code for this purpose is being written. (Another graduate research associate of the PI takes part in this work.)

\section{Plans for the next year (1992)}

(a) We plan to continue and, possibly, complete the studies of the magnetic dynamo problem of 1 . above.

(b) The PI has become aware of two significant facts concerning stability of periodic flows. First, it can be important in the physics of atmosphere (see, e.g.. A. Batchaev, Izv. Akad. Nauk., Fiz. Atmos. i Okeana 25, 1989, 434). Second, many externally forced periodic flows can be studied experimentally, by using electrically conducting fluids placed in appropriately configured magnetic fields (e.g., J. Sommeria et al., in: Mechanics of fluids and transport processes, vol. 
10, Kluwer, 1989). On the other hand, due to our progress on time dependent flows described in 2. above, it became clear that many analytical and computational problems formulated in our proposal are more ripe to be solved than it appeared at the time the proposal was written. Therefore, this part of our project calls for an accelerated research, which can be done with the help of a suitable postdoctoral research associate. There appear to be some good candidates for this task. One was mentioned above in 2. Another is Dr. A. Thess of Ecole Normale Superieure Lyon, whose thesis work on numerical investigation of periodic (time independent) flows has been recently submitted to Phys. Fluids (and reviewed by this PI). His approach is similar to one suggested in the proposal for the present project. Hence, he could easily enter the investigation of the time dependent periodic flows which we plan for the next year.

In view of the above, we need some increase of funding for the next year. It will be provided totally by the University of Alabama. The total requested amount of the DOE cost sharing does not exceed the amount which was planned for the year 1992 at the time of the grant renewal (December 1990).

(c) The studies of the films flowing down cylinders will be continued. Preliminary considerations show that regimes might be possible where the interface equation acquires a third derivative dispersive term. This could explain the nonlinear periodic waves observed in many studies starting with experiments of P. Kapitza (1948). Also, the possibility of generalizing our equation for strongly undulating films (see 4 . above) to regimes with significant role of inertia should be studied.

(d) The studies of the core-annular flow will be continued. The possibility of an evolution equations (similar to those described in 6 . above) for the case of large Reynolds numbers in the annular film will be studied. The work on numerical simulation of evolution equations will continue.

$$
\text { Clexauder trenbel }
$$

Professor Alexander Frenkel, Principal Investigator 

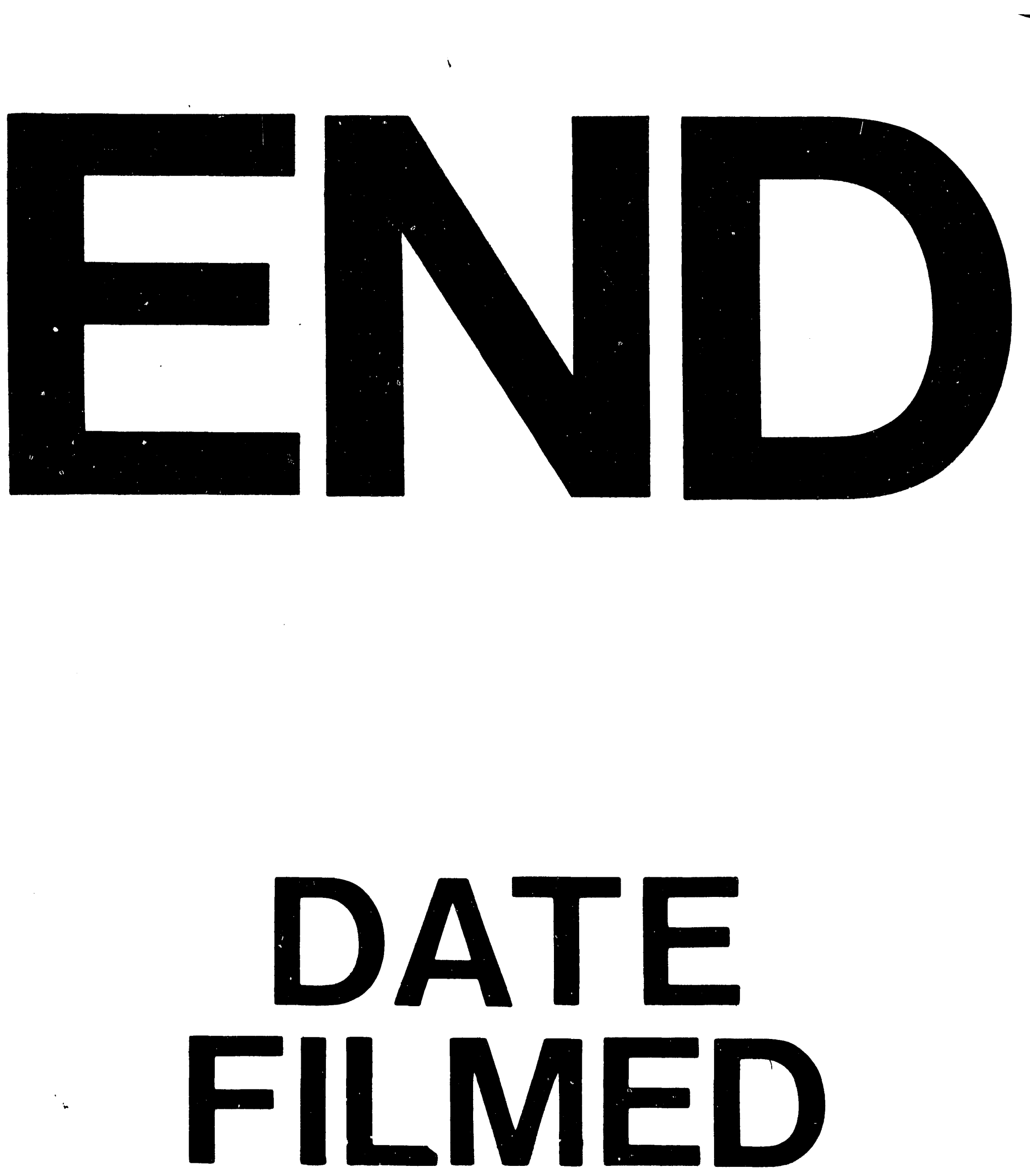

1

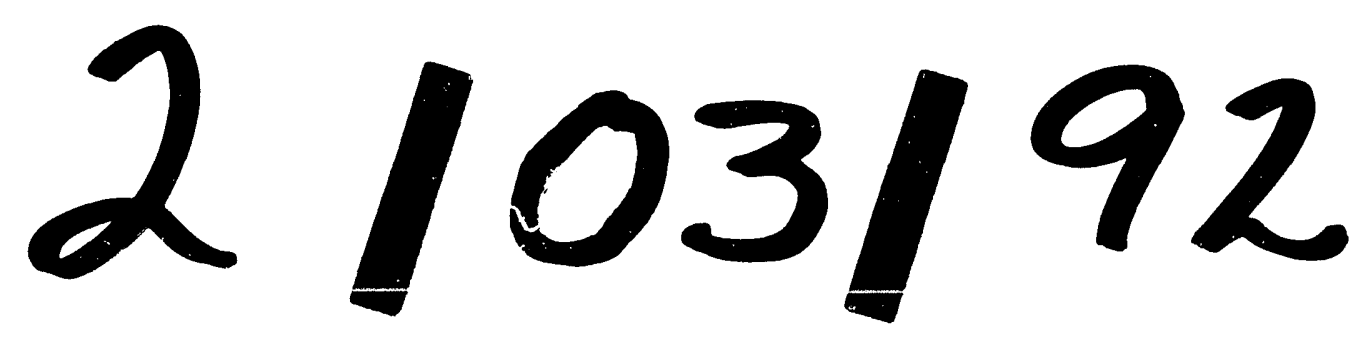


